\section{Transgastric endoscopic ultrasound with fine- needle aspiration and ERCP using percutaneous- assisted transprosthetic endoscopic therapy in a gastric bypass patient}

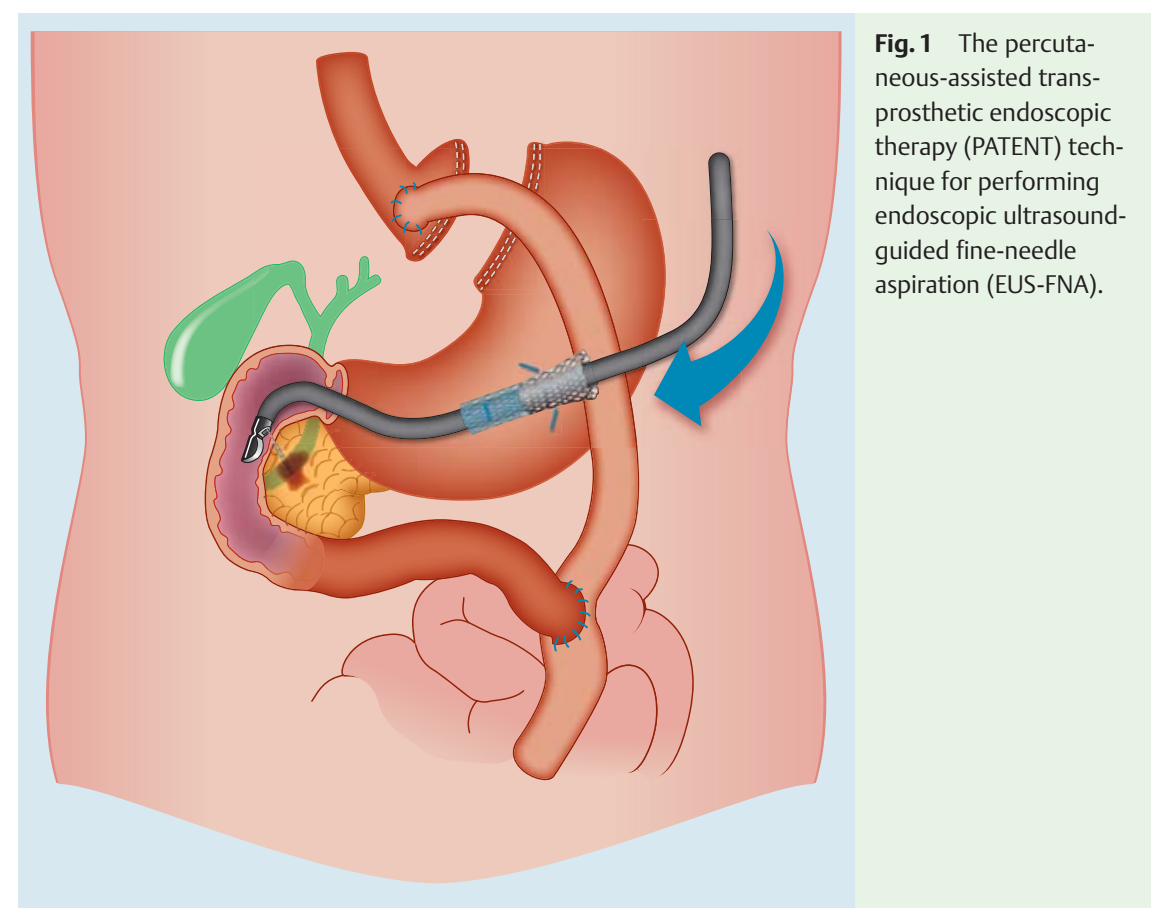

Percutaneous-assisted transprosthetic endoscopic therapy (PATENT) is a novel endoscopic technique recently developed which uses a nonnatural orifice to gain access to the gastrointestinal tract for performance of interventional procedures during a single session [1]. PATENT permits endoscopic retrograde cholangiopancreatography (ERCP) in patients with longlimb Roux-en-Y gastric bypass (RYGB) and overcomes limitations of other accessgaining techniques $[2,3]$. We present the first case of endoscopic ultrasound (EUS) with fine-needle aspiration (FNA) using PATENT.

A 66-year-old woman with prior RYGB presented for evaluation of obstructive jaundice (total bilirubin $10.1 \mathrm{mg} / \mathrm{dL}$ ). Abdominal CT demonstrated a 2-cm pancreas head mass suggestive of adenocarcinoma with intra- and extrahepatic biliary dilatation and pancreatic ductal dilatation. There was no definitive vascular invasion or encasement. Prominent lymph nodes were also noted. The patient was deemed a borderline candidate for pan- creaticoduodenectomy. Neoadjuvant chemotherapy and biliary decompression were recommended. A peroral EUS exam from the small gastric pouch showed limited views of the tumor and FNA was nondiagnostic. ERCP was attempted using a single-balloon enteroscope, but cannulation of the biliary tree was unsuccessful. Repeat ERCP and EUS were successfully performed 4 days later with the patient supine and under general anesthesia, using the PATENT technique [2,3]. After passing a single-balloon enteroscope via the mouth to the excluded stomach, a gastrostomy tract was created using the Russell introducer method and a $7 \mathrm{~cm} \times$ $18 \mathrm{~mm}$ fully covered esophageal self-expandable metal stent (SEMS) (AlimaxxES; Merit Medical Endotek, South Jordan, UT, USA) was placed across the tract. A linear-array echoendoscope (GF-UCT180; Olympus America, Center Valley, PA, USA) was passed via the gastrostomy tract through the esophageal SEMS to the excluded stomach and duodenum ( $\bullet$ Fig. 1 , - Fig.2a). Standard EUS images were ob- tained ( $\bullet$ Fig. $\mathbf{2 b}$ ) and revealed a mass abutting the portal venous confluence. FNA with rapid on-site cytopathology demonstrated adenocarcinoma. ERCP (TJF-Q180V; Olympus America) was performed with placement of a $4 \mathrm{~cm} \times 10 \mathrm{~mm}$ transpapillary uncovered biliary SEMS (Wallflex; Boston Scientific, Natick, MA, USA) ( Fig. 2c). Following ERCP the esophageal SEMS was removed by traction and a standard 26-Fr balloon-tipped gastrostomy tube was placed in the tract. Neoadjuvant therapy was followed by restaging with abdominal CT 3 months later, which suggested development of metastases. The patient is receiving palliative chemoradiation.

This case demonstrates that the PATENT approach is an option in patients with RYGB in whom biliary or pancreas head pathology is strongly suspected and EUS with ERCP is deemed necessary.

PATENT is successful when therapeutic ERCP interventions are needed in RYGB patients [2,3]. As in the present case, PATENT appears useful for diagnostic purposes. Using PATENT in our patient allowed routine EUS with diagnostic FNA cytology when an initial EUS exam was limited and yielded a nondiagnostic specimen.

Wilson et al. [4] recently identified challenges of EUS in patients with surgically altered anatomy. EUS failed to image the common bile duct and pancreas head in $86 \%$ of patients with RYGB; however, the pancreas body and tail were visible for examination and accessible for adequate FNA. Thus, routine peroral EUS appears adequate for distal pancreas lesions in RYGB patients. Nearly $70 \%$ of pancreas adenocarcinomas occur in the pancreatic head, and EUS evaluation and the ability to perform diagnostic FNA in this area are paramount [5].

\section{Endoscopy_UCTN_Code_TTT_1AS_2AD}

\section{Competing interests: None}

\section{Ryan Law ${ }^{1}$, Todd H. Baron², Mark D. Topazian'}
${ }^{1}$ Division of Gastroenterology and Hepatology, Mayo Clinic, Rochester, Minnesota, USA
${ }^{2}$ Division of Gastroenterology and Hepatology, University of North Carolina, Chapel Hill, North Carolina



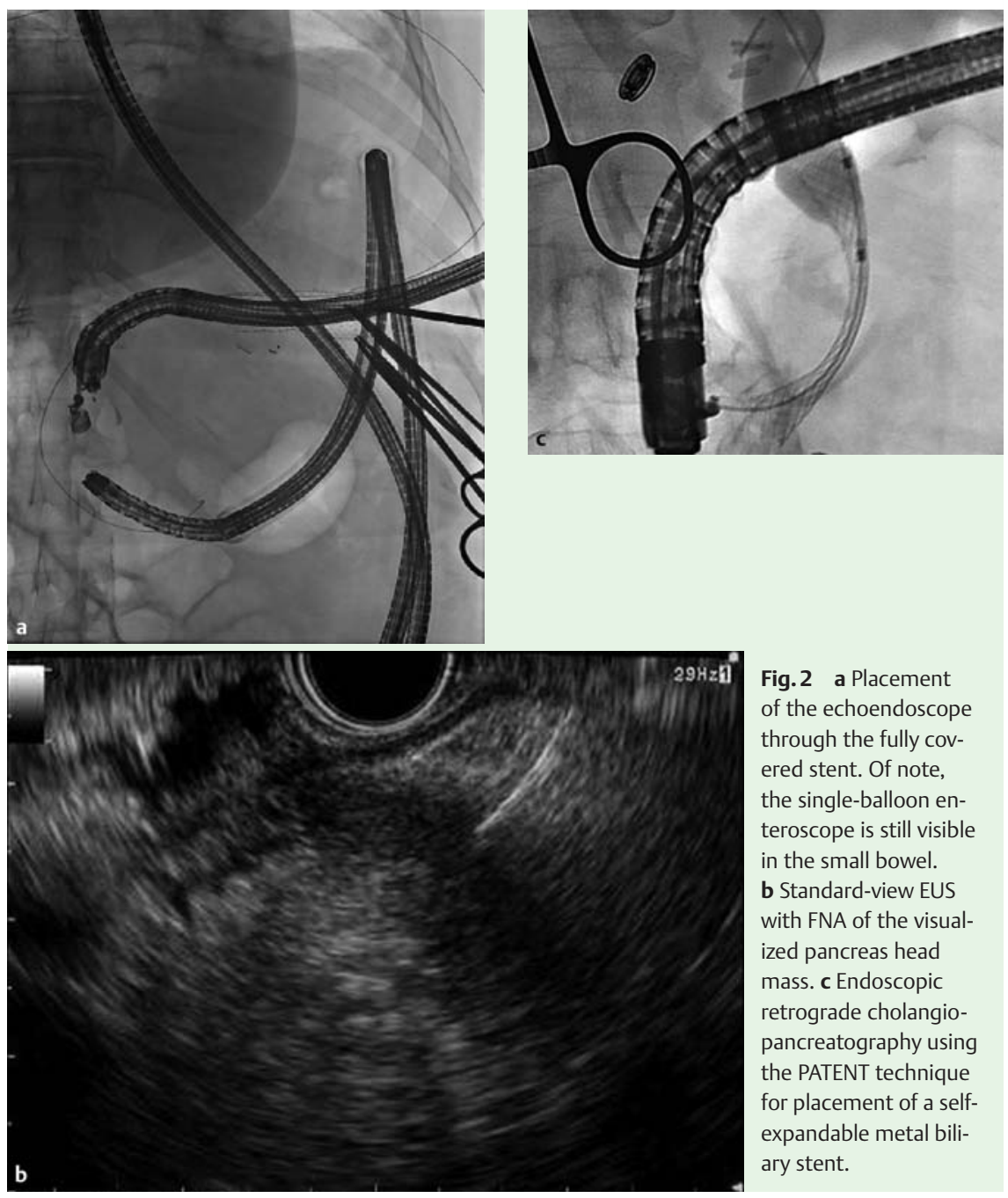

Fig. 2 a Placement of the echoendoscope through the fully covered stent. Of note, the single-balloon enteroscope is still visible in the small bowel. b Standard-view EUS with FNA of the visualized pancreas head mass. c Endoscopic retrograde cholangiopancreatography using the PATENT technique for placement of a selfexpandable metal biliary stent.

\section{References}

1 Baron TH, Song LM. Percutaneous assisted transprosthetic endoscopic therapy (PATENT): expanding gut access to infinity and beyond! (with video). Gastrointest Endosc 2012; 76: 641-644

2 Baron TH, Song LM, Ferreira LE et al. Novel approach to therapeutic ERCP after longlimb Roux-en-Y gastric bypass surgery using transgastric self-expandable metal stents: experimental outcomes and first human case study (with videos). Gastrointest Endosc 2012; 75: 1258 - 1263

3 Law R, Wong Kee Song LM, Petersen BT et al. Single-session ERCP in patients with previous Roux-en-Y gastric bypass using percutaneous-assisted transprosthetic endoscopic therapy: a case series. Endoscopy 2013; 45: 671-675

4 Wilson JA, Hoffman B, Hawes RH et al. EUS in patients with surgically altered upper GI anatomy. Gastrointest Endosc 2010; 72: 947-953

5 Modolell I, Guarner L, Malagelada JR. Vagaries of clinical presentation of pancreatic and biliary tract cancer. Ann Oncol 1999; 10 (Suppl. 04): 82-84

\section{Bibliography}

DOI http://dx.doi.org/ 10.1055/s-0034-1377281

Endoscopy 2015; 47: E104-E105

(c) Georg Thieme Verlag KG

Stuttgart · New York

ISSN 0013-726X

\section{Corresponding author}

Mark D. Topazian, MD

200 First Street SW

Rochester

55905 Minnesota

USA

topazian.mark@mayo.edu 